DOI: 10.1002/mabi.((insert number))

\title{
Communication
}

\section{Dual-Functionalization Device for Therapy through Dopamine Release and Monitoring}

Georgina Fabregat,* Alessia Giménez, Angélica Díaz, Jordi Puiggalí and Carlos Alemán*

Dr. G. Fabregat, A. Giménez, Dr. A. Díaz, Prof. Dr. J. Puiggalí, Prof. Dr. C. Alemán Departament d'Enginyeria Química (EEBE) and Barcelona Research Center for Multiscale Science and Engineering, Universitat Politècnica de Catalunya, C/ Eduard Maristany, 10-14, Ed. I2, 08019, Barcelona, Spain.

E-mail: georgina.fabregat@upc.edu and carlos.aleman@upc.edu

\begin{abstract}
A dual-functional device is fabricated to release progressively dopamine (DA) from a biohydrogel under real-time monitoring via electrochemical detection. For this purpose, a poly- $\gamma$-glutamic acid biohydrogel is assembled with a poly(3,4-ethylenedioxythiophene) (PEDOT) layer, previously deposited onto a screen printed electrode. The biohydrogel is formulated to achieve dimensional stability and maximum DA-loading capacity. Conditions for DA-loading are influenced by the oxidation of the neurotransmitter in acid environments and the poor resistance of PEDOT to the lyophilization. The performance of the device is proved in a medium with the physiological $\mathrm{pH}$ of blood and the cerebrospinal fluid. The progressive release of DA is successfully monitored by the device, the limit of detection and sensitivity of the integrated sensor being $450 \mathrm{nM}$ and $8 \cdot 10^{-5} \mathrm{~mA} / \mu \mathrm{M}$, respectively. The effect of electrochemical stimulation in the kinetics of the DA release is also investigated applying potential ramps in cyclic phase to alter the biohydrogel morphology.
\end{abstract}




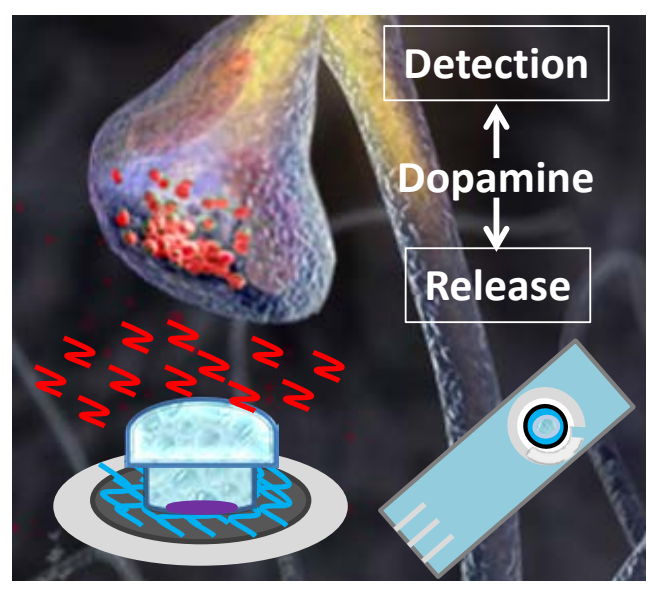




\section{Introduction}

Dopamine (DA) is a catecholamine that acts as a neurotransmitter, transmitting signals between the nerve cells of the brain and helping to regulate movement, emotional responses, pleasure, pain and motivation. The temporal fluctuation of DA concentration in the human brain has a critical effect on neurodegenerative disorders very relevant in current society, such as Parkinson's disease, Harrington's disease, bipolar disorder and attention deficit disorder. ${ }^{[1]}$ This neurotransmitter is also associated with some psychological conditions, such as psychosis and schizophrenia. ${ }^{[2]}$

Although DA can be monitored using a wide variety of techniques (e.g. spectroscopy and colorimetry), ${ }^{[3]}$ the most straightforward technique for monitoring of inherently redox active DA are the electrochemical methods, which allow its discrimination from other structurally related neurotransmitters and from interferents (e.g. uric acid in the blood and ascorbic acid in the brain). Because of the central role of DA, thousands of papers have been published during the last decades on its electrochemical detection and sensing,[3] many of them covered in recent very good reviews. ${ }^{[4]}$ On the other hand, the administration of DA can help to relieve the symptoms of some of the above mentioned disorders, as for example Parkinson's disease in which the progressive degeneration of the nigrostriatal dopaminergic system causes DA loss. ${ }^{[5]}$ Currently, oral administration of DA precursor levodopa (L-DOPA) remains the gold standard for the treatment of these disorders, even though in many cases therapeutic effects decrease over the long-term (i.e. a gradual increment of the drug dose is required) and motor fluctuation may result as a side effect of the treatment. In order to relieve the undesirable effects of L-DOPA, in the last few years a few nanomaterials-based delivery systems have been developed for the targeted release of DA (e.g. nanoparticles, ${ }^{[6]}$ carbon nanotubes, ${ }^{[7]}$ carbon $\operatorname{dots}^{[8]}$ and 3D hierarchical nanostructures ${ }^{[9]}$ ). In this work we propose an integrated bioplatform for the simultaneous detection and release of DA. From a fundamental perspective, this promising dual-functionalizing strategy offers a distinctive design principle 
for real-time monitoring of progressively released neurotransmitters and opens a window for the development of devices for theranostic therapeutics for neurodegenerative diseases.

\section{Experimental section}

Materials and Methods are available in the Supporting Information. Details about the manufacture of the different components of the dual-functionalized device are provided in the Supporting Information.

\section{Results and Discussion}

The procedure used to fabricate the device for release and detection of DA, hereafter abbreviated RD-DA, is summarized in Figure 1. The RD-DA is based on a screen printed electrode (SPE in Figure 1A) that contains a carbon working electrode (WE), a platinum auxiliary electrode (AE) and a silver reference electrode (RE). Firstly, the carbon WE with 4 $\mathrm{mm}$ in diameter is modified by poly(3,4-ethylenedioxythiophene) (PEDOT), which is obtained by anodic polymerization of 3,4-ethylenedioxythiophene (EDOT) monomers under a constant potential of $1.40 \mathrm{~V}$ (Figure 1B). The sheet-like compact structure of the bare carbon WE (Figure 2A) changes considerably after electrodeposition of the PEDOT layer. SEM micrographs of PEDOT-coated SPE show a sponge-like morphology with pores that facilitate the access and escape of electrolyte ions during electrochemical redox processes (Figures 2BC). After this, a thick (2 mm) Teflon ${ }^{\circledR}$ film with a hole of the same diameter as the WE is placed onto the modified SPE to protect both the AE and RE (Figure 1C), which are essential for the electrochemical detection process. Then, the PEDOT-modified WE is coated with a thin gelatin layer ( $1 \mu \mathrm{L}$ of $10 \mathrm{w} / \mathrm{v}$ gelatin solution) to facilitate the adhesion between the PEDOT layer and the hydrogel used for DA loading and release (Figure 1D). 
After this, a disulfide-cross-linked poly- $\gamma$-glutamic acid ( $\gamma$-PGA) hydrogel matrix is synthesized in situ onto the gelatin layer. $\gamma$-PGA is a poly( $\gamma$-peptide) derived from glutamic acid (Glu), in which peptide bonds involve the $\gamma$-carboxyl group and $\alpha$-amino groups (Figure $1 \mathrm{E})$. This compound, which is naturally synthetized as a slime layer by a variety of members of the genus Bacillus, ${ }^{[10]}$ is commercially used in cosmetics, medicine, and industrial products (e.g. water absorption agents, moisturizing agents, thickening agents, absorption enhancers of minerals, sustained release drug delivery systems, and biodegradable agglomerated detergents). ${ }^{[11]}$ In this work $\gamma$-PGA chains have been chemically cross-linked using a water soluble cystamine (WSC) as cross-linker agent, which regulates the characteristics of the resulting biohydrogel (i.e. cross-linking density, pore size, water absorption, and degradability), ${ }^{[12]}$ and ethylcarbodiimide methiodide (EDC) as condensing agent (Figure 1E). SEM micrographs displaying the PEDOT/gelatin/ $\gamma$-PGA interfaces are displayed in Figures 2D-F. The boundaries of the PEDOT film and the gelatin layer are very well defined at the corresponding interface, whereas a biphasic interface in which the $\gamma$-PGA hydrogel penetrates into the gelatin separates such two materials. The formation of this biphasic region, which is of micrometric thickness, is consequence of the diffusion of the $\gamma$-PGA chains through the gelatin layer and their subsequent reticulation during the synthesis of the biohydrogel. Besides, comparison of the pores at both the biphasic region and the external sides of the hydrogel (Figures 2D-F) with those the internal region (Figure 2G) reflects important differences. Thus, pores are significantly larger in the former zones than in the internal region since the diffusion of WSC molecules inside the gelatin and towards the external sides is limited by the own gelation reaction. Consequently, the crosslinking degree is higher at the internal region, which affects the size of the pore.

After removing the Teflon ${ }^{\circledR}$ film, the assembled device was washed with distilled water for 1 $\mathrm{h}$ to eliminate the excess of EDC and achieve the maximum swelling (Figure 1F). The morphological characteristics of the hydrogel, which were defined during the synthetic 
process through the $\gamma$-PGA:WSC:EDC ratio, and the choice of the swelling medium were critical to optimize the DA loading process in the assembled device, as is detailed below. Then, the DA was incorporated into the $\gamma$-PGA hydrogel through osmotic loading using a citric acid / sodium citrate buffer solution ( $\mathrm{pH} 4.5$ ) with $6 \mathrm{mg} / \mathrm{mL}$ DA as incubation medium (Figure 1G). This acid environment protected the DA molecule from oxidative degradation processes. Finally, the excess of DA adsorbed onto the surface of the $\gamma$-PGA hydrogel was eliminated by washing with phosphate buffered saline (PBS) solution at $\mathrm{pH} 7.2$ (Figure 1H). After this, the integrated RD-DA was ready to be used as a dual-functional device capable of monitoring the release of DA in real-time (Figure 1I).

Crucial points in this fabrication process were the selection of PEDOT as electrode material and properties of the $\gamma$-PGA matrix, especially the dimensional stability of the biohydrogel, which is related with its swelling capacity, and the DA-loading ability. The selection of PEDOT was motivated by the stability of this conducting polymer, which is significantly than that of other polyheterocycles as polypyrrole, and its ability for the selective determination of very low DA concentrations (i.e. from 60 to $500 \mathrm{nM}$ depending on the presence or not of electrocatalysts). ${ }^{[13]}$ It is worth noting that the electrochemical stability of the RD-DA device is defined by the performance of the PEDOT electrode. The stability of PEDOT for DA detection was examined by investigating the variation of the oxidation peak intensity against the number of consecutive oxidation-reduction cycles for $1 \mathrm{mM}$ DA in $0.1 \mathrm{M}$ PBS. After 25 consecutive cycles, the reduction of the peak intensity was $8 \%$ only, demonstrating the good properties of the selected conducting polymer.

On the other hand, the properties of the $\gamma$-PGA matrix strictly depend on the formulation of the prepared hydrogels. In this work seven different $\gamma$-PGA biohydrogels with different crosslinking characteristics were prepared by combining the following parameters: (i) $\gamma$-PGA average molecular weight $(\mathrm{Mw}=350,000$ and 2,000,000); (ii) the $\gamma$-PGA : WSC ratio; and (iii) the EDC : $\gamma$-PGA ratio. The synthetic procedure, which was adapted from the one 
described by Matsusaki et al., ${ }^{[14]}$ is described in the Supplementary Information. For brevity, the discussion of different observed behaviors is focused on the three hydrogels that provided better results (Table S1). These are LPGA-1 and LPGA-2, which were obtained using low molecular weight $\gamma$-PGA $(\mathrm{Mw}=350,000)$ and a $\gamma$-PGA / EDC /WSC ratio of 5 / 5 / 1.5 and 5 / 5 / 2.5, respectively, and HPGA-1 that was derived from high molecular weight $\gamma$-PGA (Mw $=2,000,000)$ using a $\gamma$-PGA / EDC /WSC ratio of $5 / 5 / 2.5$.

The swelling ratio (SR) of the hydrogels depends on both their chemical formulation and the $\mathrm{pH}$, as is described in the Supplementary Information. Considering that the pKa of noncrosslinked carboxylate groups is $\sim 2.2$, neutral solutions produced higher SRs than acid ones in all cases (Table S2), which could affect the dimensional stability of the RD-DA device (i.e. when swollen, the hydrogel could cover the area of the electrodes, AE and RE, and hinder the monitoring of released DA). However, this effect was smaller for the formulation of the LPGA-2 biohydrogel $(\mathrm{SR}=517 \% \pm 49 \%$ and $793 \% \pm 15 \%$ after $48 \mathrm{~h}$ immersed in buffers with pH 4.5 and 7.3, respectively) than for LPGA-1 and HPGA-1. The response of the LPGA-2 hydrogel after immersion in distillate water for $3 \mathrm{~h}$ is illustrated in Figure 3A. Moreover, analysis of the dynamic response of LPGA-2 against the change of $\mathrm{pH}$ (Figure 3B) reflected that the SR stabilizes much more quickly at pH $7.3(\sim 1 \mathrm{~h})$ than at $\mathrm{pH} 4.3(\sim 24 \mathrm{~h})$.

Conditions for the DA-loading into the $\gamma$-PGA hydrogel were optimized considering the following variables: (i) the formulation of the hydrogel (i.e. three selected hydrogels, Table S1); (ii) the concentration of DA in the incubation solution (i.e. 3 and $6 \mathrm{mg} / \mathrm{mL}$ ); and (iii) the $\mathrm{pH}$ of the incubation solution (i.e. $\mathrm{pH} 7.2,4.5$ and 2.2). Although DA was successfully loaded in all tested conditions, this neurotransmitter underwent a drastic oxidation when a hydrochloric acid / chloride buffer solution with pH 2.2 was used as loading medium (Figure S4). In spite of this, some hydrogel formulations showed an outstanding behavior, favoring a 
very rapid loading of DA and, subsequently, acting as protection element against oxidation processes (Figure 3C).

Finally, the medium selected for DA loading was the citric acid / phosphate buffer ( $\mathrm{pH} 4.5$ ) with $6 \mathrm{mg} / \mathrm{mL}$ of neurotransmitter. Although immersion of lyophilized hydrogels into such medium ensured a very fast loading process, unfortunately, the PEDOT layer deposited on the SPE was not strong enough to withstand the lyophilization process without deterioration. Accordingly, the DA loading was carried out by osmosis, immersing the device without lyophilizing the $\gamma$-PGA hydrogel into the selected medium during $24 \mathrm{~h}$. After this, the superficial DA was eliminated by washing the device with PBS (pH 7.2). It is worth noting that, in such conditions, UV-vis measurements showed that the concentration of DA in the residual loading medium was identical to the initial one $(6 \mathrm{mg} / \mathrm{mL})$, evidencing that this was also the amount of DA inside the hydrogel once the osmotic equilibrium was reached.

Figure 4 proves the remarkable performance of the loaded RD-DA integrated device once it immersed into the release medium, which consisted in phosphate buffer solution at $\mathrm{pH} 7.3$ (physiological pH of blood and cerebrospinal fluid). Figure 4A compares the evolution of the DA concentration potentiodynamically detected by the RD-DA system with those determined using conventional spectroscopic and electrochemical measures, hereafter denoted controls 1 and 2, respectively. As the concentration of DA in the medium increases with time, results from the controls demonstrate the release capacity of the RD-DA device. Results from control 2, which is based on cyclic voltammetry (CV) measurements using independent SPE electrodes coated with PEDOT but without hydrogel, are influenced by the high affinity of DA towards PEDOT and, in general, towards conducting polymers. ${ }^{[13,15]}$ Thus, control 2 detects the local accumulation of DA onto the surface of the electrode, which is not considered by UV-vis spectroscopic measurements, explaining the quantitative differences between the two controls (Figure 4A). This feature is corroborated by the fact that, after 50 min, such accumulation saturates and, therefore, the DA concentration detected by control 2 
does not increase. In contrast to controls 1 and 2, the RD-DA device detects the DA remaining in the hydrogel, as is evidenced by negative slope of the detection profile, reflecting that the concentration of loaded DA decreases with time. Thus, in the recorded voltammograms the peak intensity of the DA oxidation with anodic peak potential at $\sim 0.3 \mathrm{~V}$ decreases when the immersion time of the RD-DA device into the release medium increases (Figures 4B-C).

The limit of the detection (LOD) of the sensor integrated into the RD-DA device was derived from the variation of the anodic peak intensity $\left(\mathrm{i}_{\mathrm{p}}\right)$, as determined by cyclic voltammetry, against the DA concentration. The LOD expressed as 3.3 $\sigma / \mathrm{S}$, where $\sigma$ and $\mathrm{S}$ are the standard deviation of the response and the slope of the calibration plot for DA concentrations ranging from 3 to $600 \mu \mathrm{M}$ is $450 \mathrm{nM}$. The sensitivity, which corresponds to the slope of calibration plot, is defined as the minimum DA concentration in the medium required to produce a certain current density in the output voltammogram. In the case of the RD-DA device the experimentally measured sensitivity is $8 \cdot 10^{-5} \mathrm{~mA} / \mu \mathrm{M}$.

The influence of electrochemical stimulation in the DA release was explored by applying several electrode potential ramps in cyclic phase that were additional to the one used for monitoring the neurotransmitter concentration. In practice, the electrochemically stimulated RD-DA device was submitted to three consecutive redox cycles each time that DA concentration was measured, while in absence of stimulation the only redox cycle applied was the one employed for monitoring. The shape of the profiles showing the DA remaining in RDDA devices when immersed in PBS at $\mathrm{pH} 7.3$ was in general independent of the electrochemical stimulation (Figure 4D), even though the applied cyclic potential ramps clearly affected the kinetics of the release at the first $\sim 40$ hours. Thus, the application of the potential caused a significant delay in the initial release of DA from the $\gamma$-PGA biohydrogel (e.g. $50 \%$ of DA was released after 3 and $10 \mathrm{~h}$ in absence and presence of electrochemical stimuli, respectively). However, the effect of such stimuli is null after $40 \mathrm{~h}$, when around $85 \%$ 
of DA has been released. This phenomenon has been attributed to the effect of the potential ramps on the porous morphology of the LPGA-2 hydrogel matrix, which transforms into a closed structure (Figure S5). The morphological alteration induced by electrochemical degradation is possible because of the flexibility of both the $\gamma$-PGA chains and the crosslinks. Thus, conformational movements of crosslinked polymer chains result in a reduction of the free volume (i.e. the hydrogel shrink), making difficult the diffusion of DA molecules towards the release medium. In spite of the success of electrochemically stimulated assays, future improvements in the design of the RD-DA device (e.g. replacing the gelatin layer used to assemble the electrode and the biohydrogel by a surface functionalization) could help regulate DA release more efficiently. Our future work will be oriented in this direction.

\section{Conclusions}

In summary, a DA release device able to monitor the delivered neurotransmitter has been developed by assembling a conducting polymer and a biohydrogel through a gelatin intermediate layer. The design and characteristics of the RD-DA device has been optimized to preserve the detection capacity of the SPE used as substrate and dimensional stability of the biohydrogel. Moreover, the DA-loading capability of the selected $\gamma$-PGA hydrogel is very high, making the RD-DA device a promising starting point for the development of theranostic therapeutic systems for neurodegenerative diseases. Besides, simple potential ramp assays have evidenced that the RD-DA device is electrochemically responsive. Although much work is still required to use this response as a tool to strictly control the DA release, this strategy may offer an excellent opportunity for the development of a second generation of RD-DA devices. Our future work is oriented towards the achievement of electro-responsive dualfunctionalization devices.

\section{Supporting Information}


Supporting Information is available from the Wiley Online Library.

Acknowledgements: Authors thank supports from MINECO and FEDER (MAT2015-69367R and MAT2015-69547-R) and AGAURS (2017SGR359). Support for the research of C.A. was received through the prize "ICREA Academia” for excellence in research funded by the Generalitat de Catalunya.

Received: Month XX, XXXX; Revised: Month XX, XXXX; Published online:

Keywords: Drug loading; Hydrogels; Poly(3,4-ethylenedioxythiophene); Polyglutamic acid; Theranostic therapy

[1] a) P. De Deurwaerdere, G. Di Giovanni, M. J. Millan, Prog. Neurobiol. 2017, 151, 57;

b) P. De Deurwaerdere, G. Di Giovanni, Prog. Neurobiol. 2017, 151, 175; c) A. Izquierdo, J.

L. Brigman, A. K. Radke, P. H. Rudebeck, A. Holmes, Neuroscience 2017, 345, 12; d) A. H. Ashok, T. R. Marques, S. Jauhar, M. M. Nour, G. M. Goodwin, A. H. Young, O. D. Howes, Mol. Psychiatry 2017, 22, 66.

[2] N. J. Yates, Rev. Neurosci., 2016, 27, 669-687.

[3] a) Y. Cheng, J. Wu, C. Guo, X.-G. Li, B. Ding, Y. Li, J. Mater. Chem. B 2017, 5, 2524; b) S. Dutta, C. Ray, S. Mallick, S. Sarkar, R. Sahoo, Y. Negishi, T. Pal, J. Phys. Chem. C 2015, 119, 246; c) B. Zhao, Y. Li, Talanta 2018, 179, 478; d) J. J. Zhao, L. M. Zhao, C. Q. Lan, S. L. Zhao, Sens. Actuators B 2016, 223, 246; (e) D. Wen, W. Liu, A. K. Herrmann, D. Haubold, M. Holzschuh, F. Simon, A. Eychmuller, Small 2016, 12, 2439; f) P. Li, B. B. Zhou, X. M. Cao, X. H. Tang, L. B. Yang, L. Hu, J. H. Liu, Chem. Eur. J. 2017, 23, 14278.

[4] a) F. Wu, P. Yi, L. Q. Mao, Chem. Soc. Rev. 2017, 46, 2692; b) J. N. Tiwarim V. Vij, K. C. Kemp, K. S. Kim, ACS Nano 2016, 10, 46; c)T. Laurila, S. Sainio, M. A. Caro, Prog. 
Mater. Sci. 2017, 88, 499; d) K. Jackowska, P. Krysinski, Anal. Bioanal. Chem. 2013, 405, 3753; e) J.A. Ribeiro, P.M.V. Fernandes, C.M. Pererira, F. Silva, Talanta 2016, 160, 653; f) E. E. Ferapontova, Electrochim. Acta 2017, 245, 664-671.

[5] E. Garbayo, E. Ansorena, M. J. Blanco-Prieto, Maturitas 2013, 76, 272.

[6] a) R. Pahuja, K Seth, A. Shukla, R. K. Shukla, P. Bhatnagar, L. K. S. Chauhan, P. N. Saxena, J. Arun, B. P. Chaudhari, D. K. Patel, S. P. Singh, R. Shukla, V. K. Khanna, P. Kumar, R. K. Chaturvedi, ACS Nano 2015, 9, 4850; b) G. Leyva-Gomez, H. Cortes, J. J. Magana, N. Leyva-Garcia, D. Quintanar-Guerrero, B. Floran, Drug Discov. Today 2015, 20, 824.

[7] Q. Guo, H. You, X. Yang, B. Lin, Z. Zhu, Z. Lu, X. Li, Y. Zhao, L. Mao, S. Shen, H. Cheng, J. Zhang, L. Deng, J. Fan, Z. Xi, R. Li, C. M. Li, Nanoscale 2017, 9, 10832.

[8] M. S. Khan, S. Pandey, A. Talib, M. L. Bhaisare, H. F. Wu, Colloids Surf. B Bionterfaces 2015, 134, 140.

[9] H. Li, Y. Jia, A. H. Wang, W. Cui, H. C. Ma, X. Y. Feng, J. B. Li, Chem. Eur. J. 2014, 20, 499-504.

[10] a) C. B. Thome, C. G. Gómez, H. E. Noyes, R. D. Housewright, J. Bacteriol. 1954, 68, 307; b) M. Ashiuchi, T. Kamei, D. H. Baek, S. Y. Shin, M. Sung, H.H.; Soda, T. Yagi, H. Misono, Appl. Microbiol. Biotechnol. 2001, 57, 764.

A. Ogunleye, V. U. Bhat, V. U. Irorere, D. Hill, C. Williams, I. Radecka, Microbiology 2015, 161, 1.

[12] a) S. Murakami, N. Aoki, Biomacromolecules 2006, 7, 2122; b) M. Taniguchi, K. Kato, A. Shimauchi, P. Xu, K.-L. Fujita, T. Tanaka, Y. Tarui, E. Hirasawa, J. Biosci. Bioeng. 2005, 99, 130; c) M. M. Pérez-Madrigal, M. G. Edo, A. Díaz, J. Puiggalí, C. Alemán, J. Phys. Chem. C 2017, 121, 3182.

[13] a) N. F. Atta, A. Galal, E. H. El-Ads, Electrochim. Acta 2012, 69, 102; b) C. Zanardi, F. Terzi, R. Seeber, Sens. Actuators B 2010, 148, 277; c) G. Fabregat, J. Casanovas, E. 
Redondo, E. Armelin, C. Alemán, Phys. Chem. Chem. Phys. 2014, 16, 7850; d) G. Fabregat, F. Estrany, M. T. Casas, C. Alemán, E. Armelin, J. Phys. Chem. B 2014, 118, 4702; e) G. Fabregat, E. Armelin, C. Alemán. J. Phys. Chem. B 2014, 118, 4669.

[14] M. Matsusaki, H. Yoshida, M. Akashi, Biomaterials 2007, 28, 2729.

[15] a) G. Fabregat, E. Córdova-Mateo, E. Armelin, O. Bertran, C. Alemán. J. Phys. Chem. C 2011, 115, 14933; b) E. Córdova-Mateo, J. Poater, B. Teixeira-Dias, O. Bertran, F. Estrany, L. J. del Valle, M. Solà, C. Alemán. J. Polym. Res. 2014, 21, 565. 


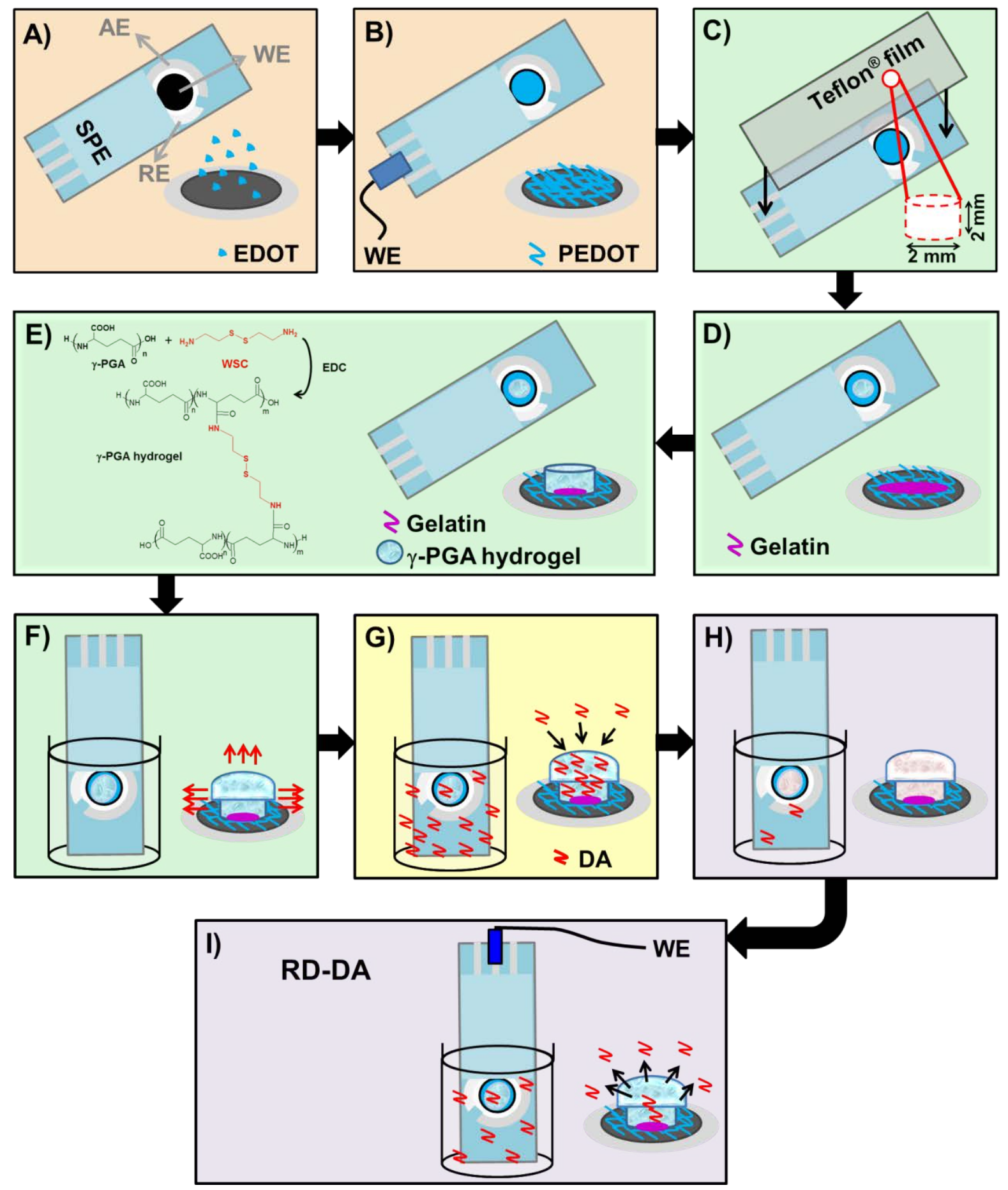

Figure 1. Schematic representation of the procedure for fabricating the integrated RD-DA (release and detection of DA) device: (A-B) Polymerization of PEDOT onto a SPE; (C-F) Assembly of the $\gamma$-PGA hydrogel matrix; (G-H) loading with DA; (I) Simultaneous release and detection of DA using the dual-functional RA-DA device. 

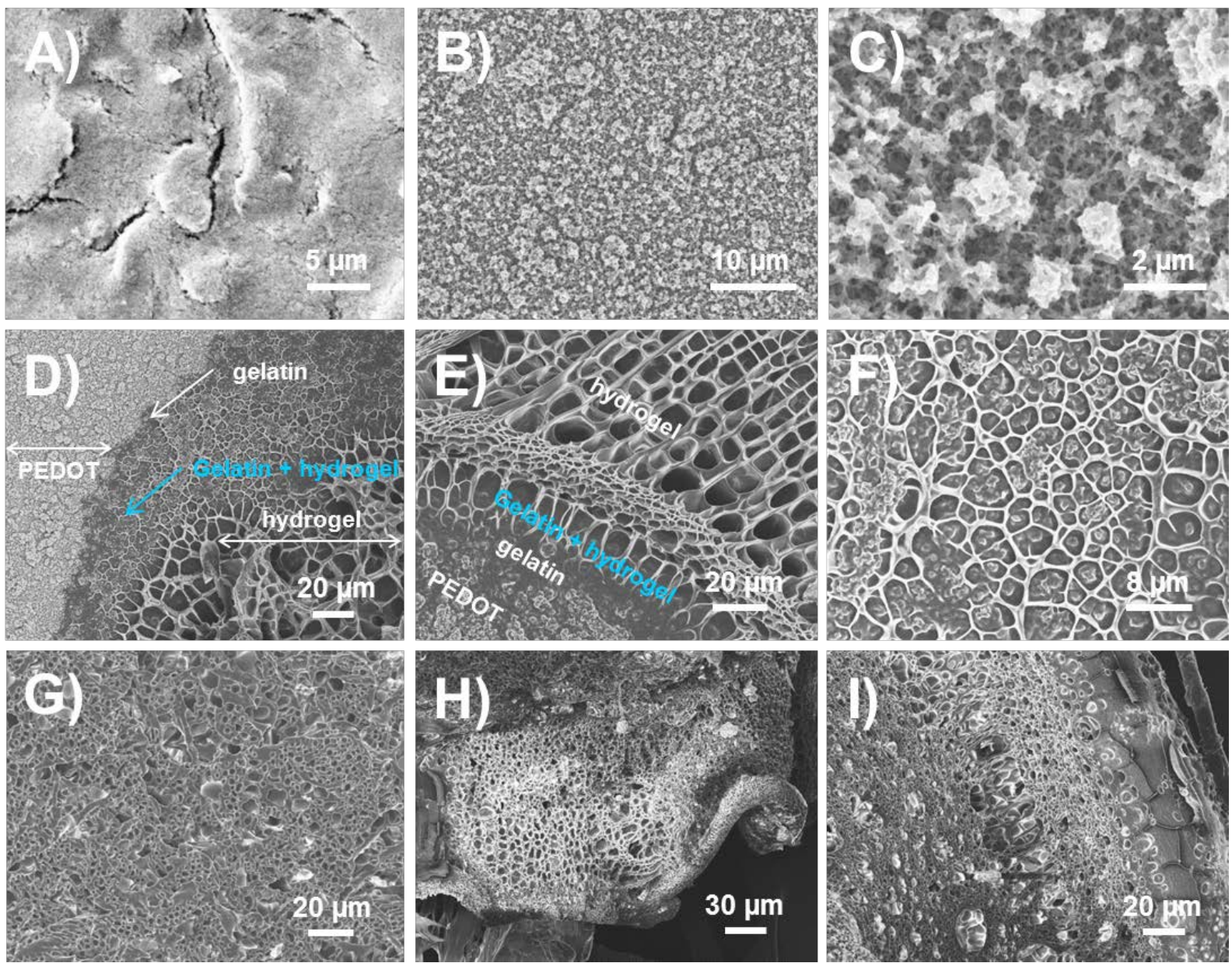

Figure 2. SEM micrographs of: (A) the carbon WE in SPE; (B and C) PEDOT-coated SPE (low and high resolution, respectively); (D-F) PEDOT/gelatin/ $\gamma$-PGA hydrogel interfaces (a representative cross section image is displayed in F); (G) interior region of the $\gamma$-PGA hydrogel; (H-I) top region of RD-DA device. In all cases the $\gamma$-PGA hydrogel corresponds to the LPGA-2 (Table S1). 
(a)
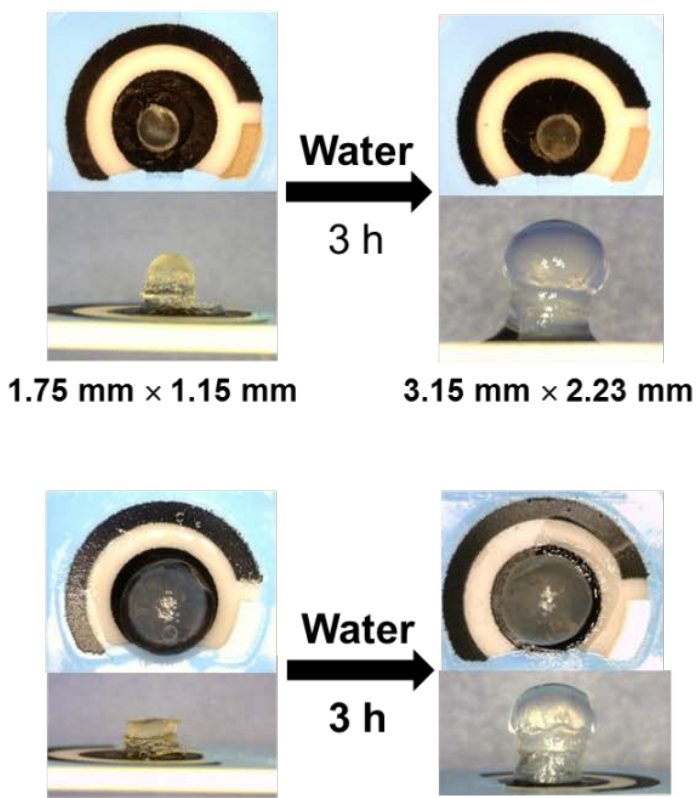

$1.80 \mathrm{~mm} \times 1.00 \mathrm{~mm}$

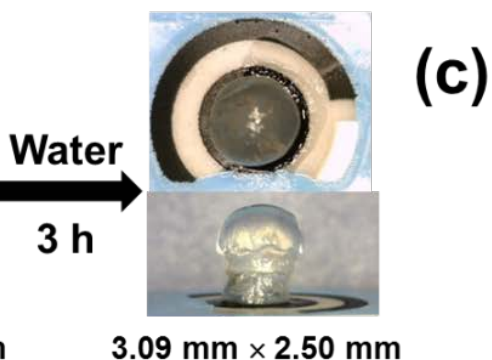

(b)
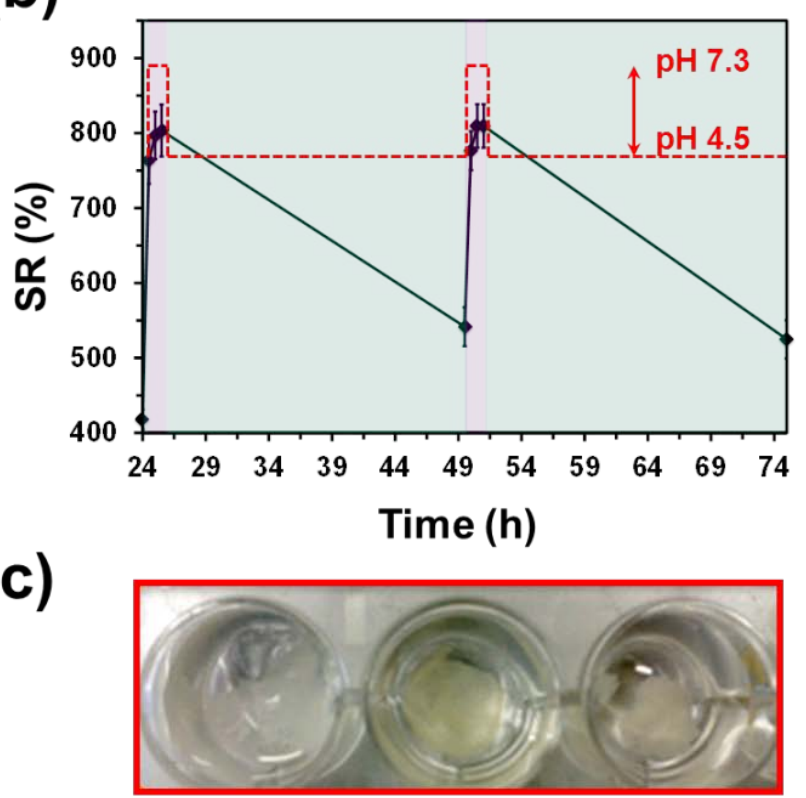

LPGA-1

$\mathrm{pH}=4.5$
LPGA-2

pH 4.5
LPGA-2

$\mathrm{pH}=\mathbf{2 . 2}$

Figure 3. (a) Change of dimensions (base $\times$ height) in two representative samples of the LPGA-2 biohydrogel, adhered to the gelatin coating the PEDOT-modified WE, after immersion in distillate water during $3 \mathrm{~h}$. (b) Swelling profile of LPGA-2, expressed as variation of the swelling ratio (SR) against time, with cyclical changes in the $\mathrm{pH}$ of the medium. Light blue regions indicate the periods of time in which the hydrogel is immersed in a phosphate buffer with $\mathrm{pH} 4.5$, while light pink regions correspond to periods of time in the phosphate buffer saline solution with $\mathrm{pH} 7.3$ (the change of $\mathrm{pH}$ is also described by red dashed line). (c) Representative experiments for DA-loading into LPGA-1 (pH 4.5) and LPGA-2 (pHs 4.5 and 2.2). Images display the aspect of the hydrogels after $4 \mathrm{~h}$ of incubation into a DA-containing (6 mg/mL) citric acid / phosphate ( $\mathrm{pH} \mathrm{4.5)}$ and hydrochloric acid / chloride (pH 2.2) buffers. 
(a)

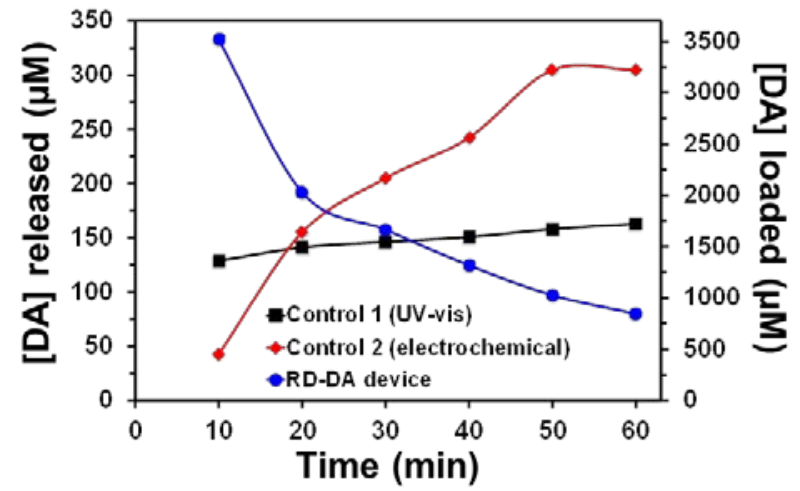

(c)

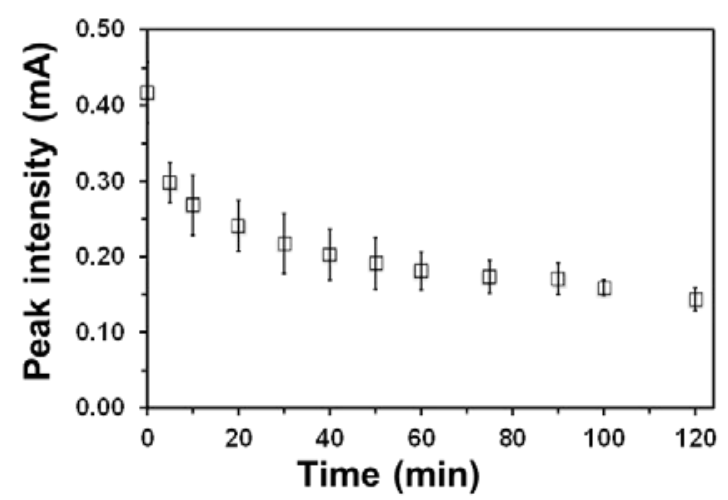

(b)

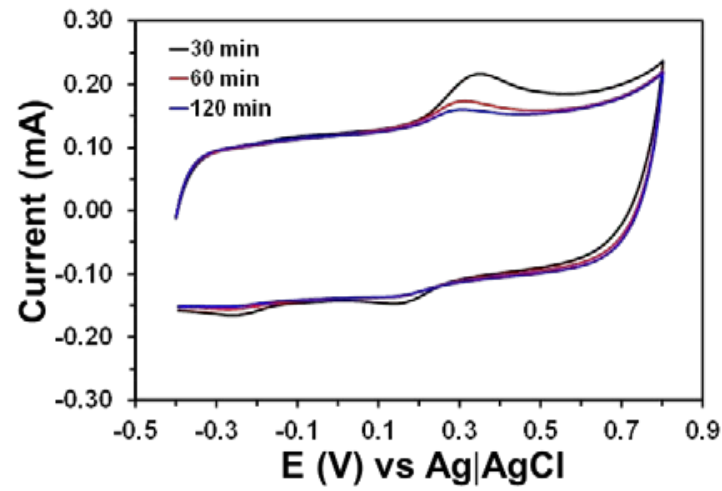

(d)

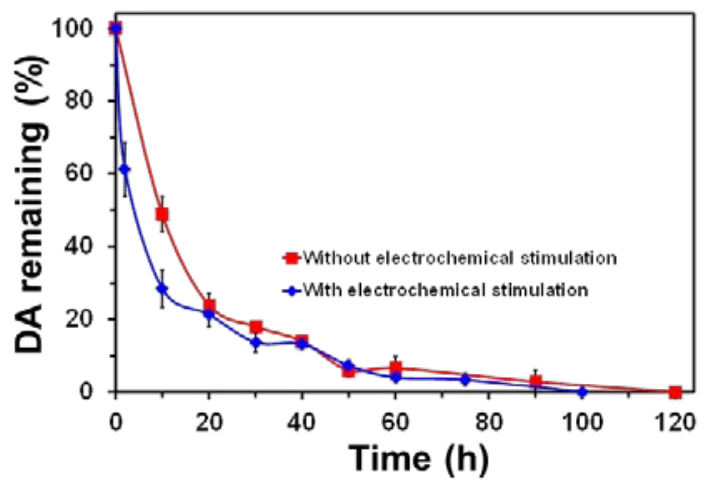

Figure 4. (a) Temporal evolution of the concentration of DA when the loaded RD-DA device is immersed into the release medium. The profiles obtained using control 1 (UV-vis spectroscopy) and control 2 (CV using a SPE coated with PEDOT) corresponds to the DA released to the bulk and to the DA accumulated onto the electrode, respectively. The profile determined using the own RD-DA device displays the progression of the neurotransmitter loaded into the hydrogel. (b) Cyclic voltammograms (scan rate: $50 \mathrm{mV} / \mathrm{s}$ ) obtained using the integrated RD-DA device after $30 \mathrm{~min}, 1 \mathrm{~h}$ and $2 \mathrm{~h}$ of immersion into the release medium. (c) Variation of the peak oxidation potential for DA at the electrode integrated into the RD-DA device against time when immersed into the release medium. (d) Effect of the electrochemical stimulation in the release profile of DA when the loaded RD-DA device is immersed in PBS at $\mathrm{pH}$ 7.3. Electrochemical stimulation consisted in the application of the three consecutive redox cycles (i.e. cyclic potential ramps changing $50 \mathrm{mV}$ per second) each time that the DA remaining in the device is monitored. 
A unique dopamine release device able to monitor continuously the delivered neurotransmitter is developed by assembling a conducting polymer and a biohydrogel. The limit of detection and sensitivity of the integrated sensor is $450 \mathrm{nM}$ and $8 \cdot 10^{-5} \mathrm{~mA} / \mu \mathrm{M}$, respectively.

G. Fabregat,* A. Giménez, A. Díaz, J. Puiggalí, C. Aleman*

Dual-Functionalization Device for Therapy through Dopamine Release and Monitoring

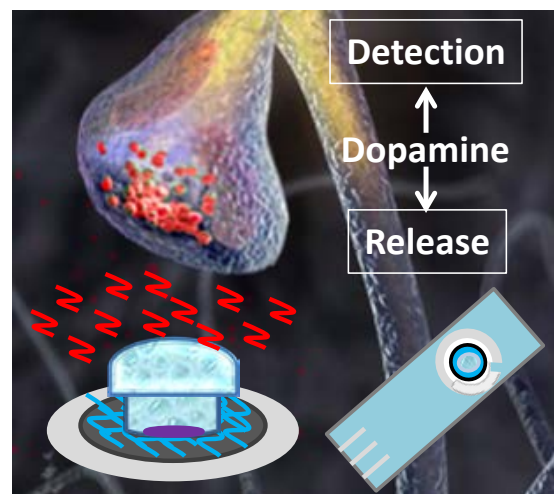


- 19 - 